\title{
INTERFERON LAMBDA WITH REMDESIVIR AS A POTENTIAL TREATMENT OPTION IN COVID-19
}

\author{
Ishag Adam $^{1} \odot$, Lukasz Szarpak², Krzysztof Jerzy Filipiak ${ }^{3}$, Jacek Smereka ${ }^{4}$, \\ Marek Dabrowski ${ }^{5} \odot$, Saeid Ghavami ${ }^{6,7} \odot$, Milosz Jaguszewski ${ }^{8} \odot$ \\ 'Department of Obstetrics and Gynecology, Unaizah College of Medicine and Medical Sciences, Qassim University, Unaizah, Saudi Arabia \\ ${ }^{2}$ Comprehensive Cancer Center in Bialystok, Poland \\ ${ }^{3}$ First Chair and Department of Cardiology, Medical University of Warsaw, Warsaw, Poland \\ ${ }^{4}$ Department of Emergency Medical Service, Wroclaw Medical University, Wroclaw, Poland \\ ${ }^{5}$ Chair and Department of Medical Education, Poznan University of Medical Sciences, Poznan, Poland \\ ${ }^{6}$ Research Institute in Oncology and Hematology, Cancer Care Manitoba, University of Manitoba, Winnipeg, Canada \\ ${ }^{7}$ Cellular and Molecular Research Center, Department of Anatomy, Faculty of Medicine, Iran University of Medical Sciences, Tehran, Iran \\ ${ }^{8} 1^{\text {st }}$ Department of Cardiology, Medical University of Gdansk, Gdansk, Poland
}

KEY WORDS: remdesivir, interferon lambda, COVID-19, SARS-CoV-2, treatment

Disaster Emerg Med J 2020; 5(3): 174

\section{Dear Editor,}

we read the article by Grein et al. [1] published in New England Journal of Medicine with interest. The new SARS-like coronavirus (now named SARS-CoV-2) that emerged in December 2019 has been shown to be closest related $(\sim 88 \%)$ to two bat-derived SARS-like CoVs (bat-SL-CoVZC45 and bat-SL-CoVZXC21), with 79\% overall sequence identity to SARS-CoV and $\sim 50 \%$ to MERS-CoV [2]. Remdesivir is well known in antiviral treatment of coronaviruses (SARS, MERS) [3], hence its consideration for SARS-CoV-2 therapy. However, we must remember that the coronavirus induces the endogenous expression of IFN- $\lambda$ and/or blocks IFN- $\lambda$, affecting inflammatory responses and mechanisms of tissue damage and repair. The main function of IFN- $\lambda$ is to prevent viral infection by establishing an antiviral state and, if infected, to slow down viral replication and dissemination. IFN- $\lambda$ acted as a unique immunomodulatory agent by modifying transcriptional and non-translational neutrophil responses, which might permit a controlled development of the inflammatory process [4]. In vitro, treatment with IFN- $\lambda$ showed potency against a variety of viruses, including SARS-CoV- 1 and MERS$\mathrm{CoV}[5]$, and currently pegylated IFN- $\lambda 1$ (peg-IFN- $\lambda 1$ ) is the only IFN- $\lambda$ currently available as a therapeutic agent.

In summary, to increase the therapeutic effect, it is therefore worth considering combined treatment of COVID-19 patients by using interferon lambda with Remdesivir.

\section{Conflict of interest}

The authors declare no conflict of interest.

\section{REFERENCES}

1. Grein J, Ohmagari N, Shin D, et al. Compassionate Use of Remdesivir for Patients with Severe Covid-19. N Engl J Med. 2020 [Epub ahead of print], doi: 10.1056/NEJMoa2007016, indexed in Pubmed: 32275812.

2. Malik YS, Sircar $S$, Bhat $S$, et al. Emerging novel coronavirus (2019-nCoV)-current scenario, evolutionary perspective based on genome analysis and recent developments. Vet Q. 2020; 40(1): 68-76, doi: 10.1080/01652176.2020.1727993, indexed in Pubmed: 32036774.

3. Gordon CJ, Tchesnokov EP, Woolner E, et al. Remdesivir is a direct-acting antiviral that inhibits RNA-dependent RNA polymerase from severe acute respiratory syndrome coronavirus 2 with high potency. J Biol Chem. 2020; 295(20): 6785-6797, doi: 10.1074/jbc.RA120.013679, indexed in Pubmed: 32284326.

4. Hemann EA, Schwerk J, Savan R. IFN- $\lambda$ 'guts' neutrophil-mediated inflammation. Nat Immunol. 2017; 18(10): 1061-1062, doi: 10.1038/ ni.3834, indexed in Pubmed: 28926532.

5. Mordstein $M$, Neugebauer $E$, Ditt $V$, et al. Lambda interferon renders epithelial cells of the respiratory and gastrointestinal tracts resistant to viral infections. J Virol. 2010; 84(11): 5670-5677, doi: 10.1128/ JVI.00272-10, indexed in Pubmed: 20335250. 